\title{
Collective Dynamics of Interacting Molecular Motors
}

\author{
O. Campàs, ${ }^{1,2}$ Y. Kafri, ${ }^{1,3}$ K. B. Zeldovich, ${ }^{4}$ J. Casademunt, ${ }^{2}$ and J.-F. Joanny ${ }^{1}$ \\ ${ }^{1}$ Institut Curie, UMR CNRS 168, 26 rue d'Ulm 75248 Paris Cedex 05, France \\ ${ }^{2}$ Departament d'ECM, Universitat de Barcelona, Avinguda Diagonal 647, E-08028 Barcelona, Spain \\ ${ }^{3}$ Physics Department, Technion, Haifa 32000, Israel \\ ${ }^{4}$ Department of Chemistry and Chemical Biology, Harvard University, 12 Oxford Street, Cambridge, Massachusetts 02138, USA
}

(Received 30 November 2005; published 19 July 2006)

\begin{abstract}
The collective dynamics of $N$ interacting processive molecular motors are considered theoretically when an external force is applied to the leading motor. We show, using a discrete lattice model, that the force-velocity curves strongly depend on the effective dynamic interactions between motors and differ significantly from those of a simple approach where the motors equally share the force. Moreover, they become essentially independent of the number of motors if $N$ is large enough $(N \geqslant 5$ for conventional kinesin). We show that a two-state ratchet model has a very similar behavior to that of the coarse-grained lattice model with effective interactions. The general picture is unaffected by motor attachment and detachment events.
\end{abstract}

\section{DOI: 10.1103/PhysRevLett.97.038101}

The collective behavior of molecular motors plays a crucial role in many biological phenomena ranging from intracellular and intraflagellar transport to axonal transport [1]. Molecular motors are often classified according to their processivity [2]. Processive motors rarely unbind from the track on which they are moving; they perform best when working in small groups and are therefore referred to as "porters". Nonprocessive motors unbind from the track frequently; they work best in large groups and are referred to as "rowers". Examples of porters are kinesin motors which move along microtubules, while classical myosin motors which move along actin filaments are examples of rowers [2].

The classification of motors into porters and rowers is based on their behavior when connected to a rigid or elastic cargo. The strong coupling between processive motors leads to an effective friction which results from motors which cannot move because other motors are bound to the track [2,3]. A strong coupling between the motors indeed exists for a microtubule pushed by kinesin motors that are bound to a surface [1]. It is also important for describing myosin motors acting in skeletal muscles. The abundance of such systems has inspired several theoretical studies of the collective behavior of strongly coupled motors $[4,5]$.

In many cases, however, this description in terms of rowers and porters is not adequate since the motors are not rigidly attached to the cargo. An important class of systems where this happens is when motors, such as kinesin, move along microtubules carrying a load which is a lipid membrane, a ubiquitous situation in living cells. This occurs, for example, when kinesins or dyneins carry a vesicle along a microtubule [1]. Recent experiments have also shown that kinesin motors moving along a microtubule act collectively to pull membrane tubes from a vesicle [6].

In this Letter, we study theoretically the collective behavior of $N$ processive motors pulling a tube out of a
PACS numbers: 87.16.Ac, 05.40.-a, 87.10.+e, 87.16.Nn

membrane and acting against the force needed to extract it [7]. A fluid membrane can only exert a force on the motors at the leading edge of the tube where the normal to the surface has a component in the direction of motor motion. For simplicity we assume here that all the force is transmitted to the leading motor.

We consider the collective behavior of the motors as a function of the applied force, $F$, the number of motors, $N$, and the effective interactions (defined as the combined effect of the microscopic details of the system on the transition rates in a coarse-grained description) between the motors. We show that the force-velocity curve $V_{N}(F)$ strongly depends on the interactions between motors and differs significantly from the mean-field treatment where independent motors equally share the force, leading to $V_{N}(F)=V_{1}(F / N)$. Moreover, we find that beyond a certain number of motors, the force-velocity curves are all indistinguishable for practical purposes. The analysis is first carried out assuming that motors do not detach from the filament. We then use numerics to show that under experimentally relevant conditions our results are not modified even in the presence of force-dependent motor detachment. Finally, we explore how the effective transition rates in the coarse-grained description arise from a more microscopic two-state model.

In a coarse-grained description of the system we first model the motors as interacting biased random walkers moving along a one-dimensional lattice $[8,9]$. The physical picture presented below strongly suggests that our conclusions will remain largely unchanged for more realistic systems where the motors can use several tracks. We first assume that the motors are fully processive and never unbind from the filament that acts as a track. All lengths are expressed in units of the lattice constant $l$ (filament period). Each site can be occupied by one motor at most, which can move to a neighboring site if empty. We label the motors with an index $\mu=1, \ldots, N$, with 1 labeling the 
leading motor on which the force is exerted. The dynamics of the motors is specified by the hopping rates defined in Fig. 1, where the boxes represent sites on the lattice and a ball with index $\mu$ indicates that the site is occupied by motor $\mu$.

The model is a generalization of the disordered exclusion model introduced in [10], which includes modifications of the rates due to nearest-neighbor interactions between the motors. The hopping rates are $p_{\mu}=p, v_{\mu}=$ $v, q_{\mu}=q$, and $u_{\mu}=u$ for $\mu \geq 2$, whereas the rates of the leading motor $(\mu=1)$ depend on the external force [11]. According to Kramers' rate theory [12], we write $p_{1}=$ $p e^{-f \delta}, q_{1}=q e^{f(1-\delta)}$, and $v_{1}=v e^{-f \delta}$, where $f$ is the force in units of $k_{B} T / l\left(k_{B} T\right.$ being the thermal energy). The dimensionless parameter $0<\delta<1$ characterizes the position of the energy barrier between two neighboring lattice sites. Attractive effective interactions correspond to reduced hopping rates $(v<p, u<q)$ and repulsive effective interactions to increased hopping rates $(v>p, u>q)$. We refer to the case $v=p$ and $u=q$ as neutral.

We first consider a system with two motors. That can be solved exactly in the long-time limit. For any finite force the probability of finding the motors $k$ sites apart decays as $\left[\left(p_{1}+q\right) /\left(p+q_{1}\right)\right]^{k}$. The average number of sites between the two motors is therefore finite and decreases with the force. Since the motors cannot overtake each other, their velocities are equal and read:

$$
V_{2}=\frac{v_{1}(p-q)+u\left(p_{1}-q_{1}\right)}{\left(v_{1}+u\right)+(p-q)-\left(p_{1}-q_{1}\right)} .
$$

For comparison, the velocity of a single motor within this model is $V_{1}=p_{1}-q_{1}$. The stall force, defined as the force for which the velocity vanishes, is $f_{s}(1)=\ln (p / q)$ for a single motor, while using Eq. (1) the stall force of two motors is

$$
f_{s}(2)=\ln \left(\frac{p v}{q u}+\frac{p}{q}-\frac{v}{u}\right)
$$

The stall force is not necessarily twice the stall force of a single motor. It is a function of the rates ratio $v / u$, which depends on the interactions between the motors, and can be either larger or smaller than $2 f_{s}(1)$ depending on whether $v / u>p / q$ or $v / u<p / q$, respectively.

The velocity $V_{2}$ is plotted for various values of $v$ and $u$ in Fig. 2 where, for clarity, we set $v / u=p / q$. The general shape of the force-velocity curve is highly sensitive to the

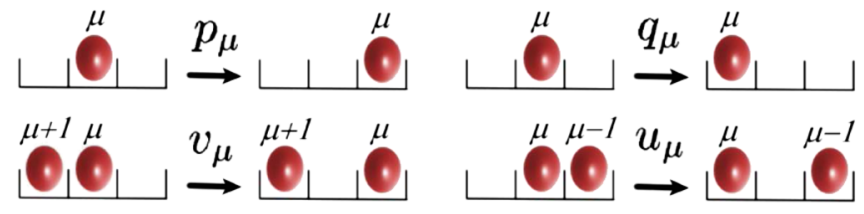

FIG. 1 (color online). Possible motor transitions and associated rates. The boxes and balls represent lattice sites and motors, respectively. interactions. For strong enough attractive interactions the velocity of two motors is smaller than that of a single motor up to a certain value of the force, at which the two motors become faster than a single motor. An experimental signature of this type is a clear demonstration of attractive interactions between the motors.

We now turn to the general case with $N$ motors. Using the results of [10], an exact expression of the velocity can be obtained in the neutral case where $v=p$ and $u=q$ on a ring geometry. In the limit where the number of vacancies in front of the first motor $(\mu=1)$ is infinite, the periodic boundary conditions do not influence the results. Building on the results of Ref. [10] one finds

$$
V_{N}=p \frac{\left[1-e^{f}(q / p)^{N}\right][1-q / p]}{e^{f \delta}[1-q / p]+e^{f}\left[q / p-(q / p)^{N}\right]} .
$$

In the neutral case, for any number of motors $f_{s}(N)=$ $N f_{s}(1)$. The force-velocity curves are shown in Fig. 3(a) for various numbers of motors. Even in the neutral case, the force-velocity curve is significantly different from the prediction $V_{N}(F)=V_{1}(F / N)$ for motors equally sharing the force. In the absence of force, the velocity is independent of the number of motors. The slope of the forcevelocity curve for vanishing forces is negative and converges exponentially fast with $N$ to $-(1-q / p)[q+(p-$ $q) \delta$. The larger the number of motors the smaller the absolute value of the slope. In particular, these results imply that for any $N \gg 1+1 / \ln (p / q)$ the force-velocity curves are almost indistinguishable [Fig. 3(a)]. For large $N$ and $f$ the motors form a dense cluster which can move only through the exponentially slow (in $N$ and $f$ ) process of a vacancy entering at one end of the cluster and exiting at the other.

We have performed continuous time Monte Carlo simulations (see e.g., [13]) to test the effect of interactions between motors. Similarly to the case of two motors, the

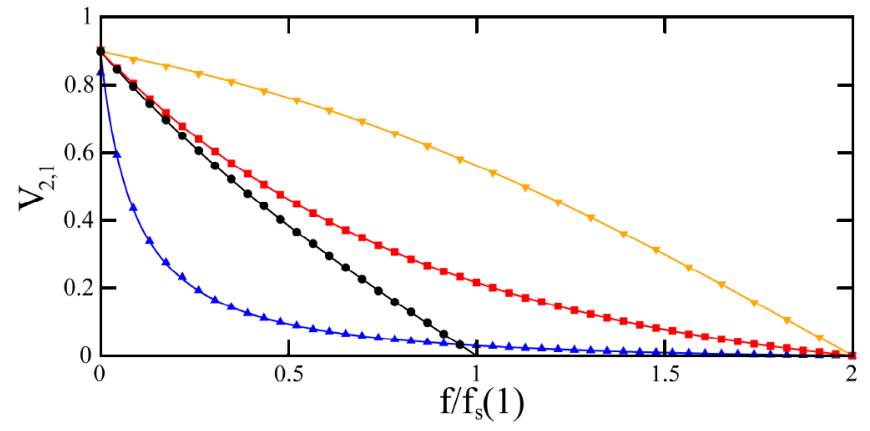

FIG. 2 (color online). Force-velocity curves of 2 motors for attractive, repulsive, and neutral interactions. Analytical solutions of Eq. (1) (solid lines) and Monte Carlo simulations (symbols). For all cases $p=1.0, q=0.1$, and $\delta=0.5$. The rates $v$ and $u$ are: $v=0.1,1.0,10.0$, and $u=0.01,0.1,1.0$ for attractive (triangle up), neutral (square), and repulsive (triangle down) interactions, respectively. The force-velocity curve for a single motor is also plotted for comparison (circle). The ratio $v / u=p / q$ so that $f_{s}(2)=2 f_{s}(1)$. All rates are in units of $p$. 

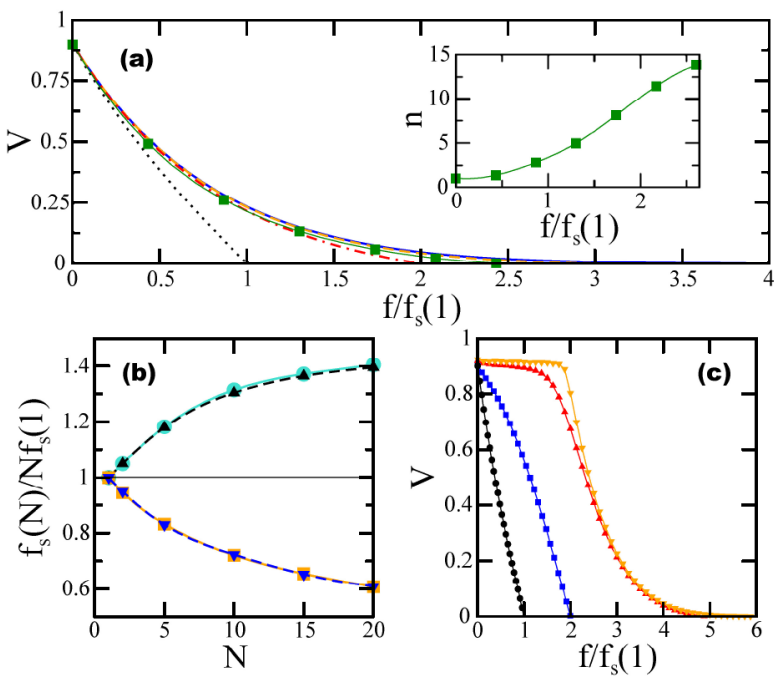

FIG. 3 (color online). (a) Force-velocity curves for the neutral case [Eq. (3)] and 1 (dotted line), 2 (dashed-dotted line), 3 (dashed line), and 100 (continuous line) motors. The forcevelocity curve obtained by numerical simulation in the presence of detachment and attachment kinetics is also shown (squares). The inset shows the evolution of $n$ with the force; $p=v=1$, $q=u=0.1, \delta=0.5, a_{0}=0.01, d_{0}=5.6 \times 10^{-3}, \delta_{d}=0.16$ [17,18]. (b) Stall force as a function of $N$ for various types of interactions. Both for attractive ( $v=0.7, u=0.5$; circles) and repulsive ( $v=1.54, u=1.1$; triangles up) interactions, the value of the stall force, $f_{s}(N)$, is the same and larger than $N f_{s}(1)$ as $v / u=1.4>p / q$. When $v / u=1.1<p / q, f_{s}(N)<$ $N f_{s}(1)$ and it has also the same value for both attractive $(v=$ $0.55, u=0.5$; squares) and repulsive ( $v=1.21, u=1.1$; triangles down) interactions. (c) Force-velocity curves in the case of repulsive interactions between highly biased motors $(p=1$, $q=0.1, v=10, u=1$ ) for 1 (circles), 2 (squares), 5 (triangles up), 10 (triangles down) motors; $\delta=0.5$. All rates are in units of $p$.

stall force is only a function of the rate ratios $p / q$ and $v / u$ [Fig. 3(b)]. When $v / u=p / q$ the stall force always satisfies $f_{s}(N)=N f_{s}(1)$ as expected. Similarly to the neutral case, for any given type of interaction the force-velocity curves are all nearly identical above a certain number of motors. In the presence of repulsive interactions, the velocity $V_{N}$ is always larger than the velocity of 1 motor and the force-velocity curves are indistinguishable if $N \geqslant 7$ $[p / q=10$; Fig. 3(c)]. In the presence of strong enough attractive interactions the velocity is smaller than that of a single motor for small forces but becomes larger at larger forces, as in the case of two motors. The force-velocity curves collapse if $N \gtrsim 5(p / q=10)$. Experimentally, one should expect a force-velocity curve independent of the number of motors if a few motors act collectively. The comparison between the velocities of many and one motor could serve as an experimental test to sort out attractive from repulsive interactions. Note that the observed stall force could be much smaller than the theoretical stall force since for many motors the velocity reaches negligible values way below stall force.
We now study the influence of the motor processivity by including the attachment and detachment kinetics in the simulations. We introduce an unbound state with a constant motor density, so that motors can attach to any site (behind the leading motor) at a constant rate, $a_{0}$. The motors bound to the filament unbind at a constant rate $d_{0}$, except for the leading motor, which detaches with a force-dependent rate $d=d_{0} \exp \left(f \delta_{d}\right)$ (where $\delta_{d}$ is a length characterizing the activated process). The number of motors in the system is not fixed but there is always a cluster of consecutive motors that forms dynamically behind the leading motor. The average number of motors in the cluster, $n$, depends on the applied force. In Fig. 3(a) we compare the forcevelocity curves in the presence and absence of attachment and detachment kinetics for the neutral case. Detachment events do not modify the force-velocity curve, as long as the number of motors clustered behind the leading motor remains large enough [Fig. 3(a), inset]. While the stall force is substantially reduced due to detachment, the apparent stall force, defined as the force for which the velocity becomes negligible, remains unchanged. For both neutral and attractive interactions, the effect of detachment on the force-velocity curves is negligible. Detachment events can significantly affect the force-velocity curve only for strongly repulsive interactions at large forces [14].

In the previous discussion, the nature of the effective interaction between motors was assumed a priori. Twostates models [15] [Fig. 4(a), inset] consider, at a coarsegrained level, some of the internal states of the motor and allow for a more detailed analysis of the effective interactions. We now show that these models are consistent with the coarse-grained lattice description with non-neutral interactions at long times and large length scales. In the strongly bound state (1) the motor feels the sawtooth potential, $W_{1}(x)$, with a period $l$, an amplitude $5 k_{B} T$ and a short segment of the sawtooth of length $a=0.2 l$. In the weakly bound state (2) the potential $W_{2}(x)$ is constant. The motors change from state 1 to state 2 and vice versa with local excitation rates $\omega_{1}(x)$ and $\omega_{2}(x)$, respectively. The
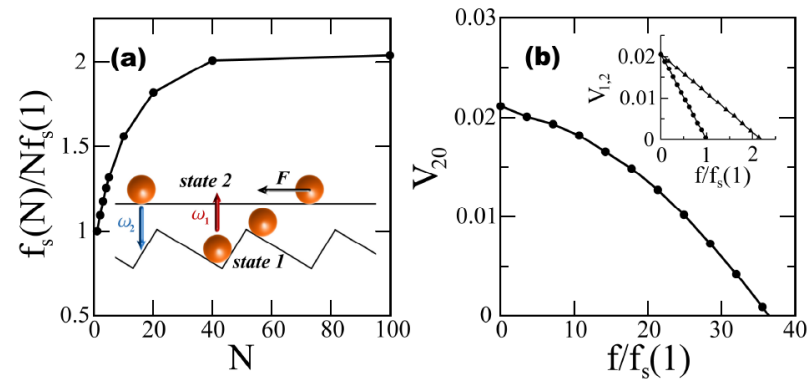

FIG. 4 (color online). Simulation results for motors with two internal states (two-state model) and only excluded volume interactions. (a) Stall force as a function of the number of motors. (b) Force-velocity curve for 20 motors. The inset in (a) sketches the two-state model, and the one in (b) shows the force-velocity relation for 1 (circles) and 2 (triangles up) motors. 
transition rates, in arbitrary units, are given by $\omega_{1}(x)=$ $\frac{\Omega}{\alpha \sqrt{\pi}} \exp \left[-(x \bmod l)^{2} / \alpha^{2}\right]$ with $\Omega=2, \alpha=0.05 l \ll a \ll$ $l$ and $\omega_{2}(x)=0.2$. We assume only hard core interactions between the motors. The repulsive potential chosen is the shifted repulsive part of a Lennard-Jones potential vanishing at $y>2^{1 / 6} \sigma=1.68 l$ ( $y$ being the distance between the motors), with an amplitude $\varepsilon=0.05 k_{B} T$. The interaction range is $\sigma=1.5 \mathrm{l}$. We have carried out simulations with a wide range of $\sigma$, from values smaller than the lattice constant to those presented here with no change in the qualitative nature of the results. We have also verified that our results remain qualitatively the same upon changing the details of the model.

We perform Langevin dynamics simulations for the motors. The equations for motor $\mu$ in state $s_{\mu}$ read

$$
\xi \frac{d x_{\mu}}{d t}=-\frac{d W_{s_{\mu}}\left(x_{\mu}\right)}{d x}-\frac{d}{d x_{\mu}} \sum_{\kappa \neq \mu} U\left(x_{\mu}-x_{\kappa}\right)+F \delta_{\mu, 1}+\eta,
$$

where $F$ is the external opposing force, and $\xi=50$ is the dimensionless friction coefficient of the motor. The random force is described by the noise term $\eta=r \xi \sqrt{\frac{6 k_{B} T}{\xi d t}}$, where $r$ is a random number taken from a uniform distribution from -1 to 1 . These equations are coupled to standard Monte Carlo steps for the transitions between the bound states 1 and 2 . Throughout the simulation, we follow the position of the first motor and determine its velocity at long times.

The force-velocity curve obtained from the simulations for 20 motors is plotted in Fig. 4(b). Since the parameters were chosen so that the stall force of one motor is small, the force-velocity relation is nearly linear for a small number of motors [Fig. 4(b), inset]. Increasing the number of motors reveals the nonlinearities. The comparison between the general shape of the curve for 20 motors [Fig. 4(b)] with the ones obtained from the coarse-grained model, suggests that the existence of two internal states for the motors leads to effective repulsive interactions. The stall force is plotted in Fig. 4(a) as a function of the number of motors. For a given $N$, it is larger than $N f_{s}(1)$, indicating that the effective rates for forward and backward movement in the equivalent coarse-grained model are such that $v / u>p / q$. As the number of motors is increased this effect becomes more important and saturates for large $N$. Overall, these results are consistent with those obtained from the lattice model with effective repulsive interactions between the motors.

The present study provides several results that can be quantitatively compared to experiments where a bead is exerting a force on a single motor moving in front of several other motors. The comparison between such experiments and our results would allow the determination of the effective interactions between motors. Moreover, our treatment is a reasonable approximation for kinesin motors carrying a vesicle subject to the friction forces that arise from its motion in the crowded environment of the cell. Any estimation of the number of motors pulling a vesicle from the force-velocity curve is questionable because of the collapse of the force-velocity curves [16].

In conclusion, we have shown, using various models of molecular motors, that the collective behavior of motors pulling on fluid membranes depends on their dynamic interactions and is very different from both the predictions of a model where the motors share equally the force, and from the behavior of strongly coupled motors.

We acknowledge stimulating discussions with M.R. Evans and the financial support of the European Commission (No. HPRN-CT-2002-00312), the Spanish M.E.C. (O.C.), MCyT (Spain), Project No. BQU200305042-C02-02, and the H.F.S.P. (Y.K. and K. B.Z.). Y. K. also thanks the Newton Institute at Cambridge UK where part of this work was carried out.

[1] J. Howard, Mechanics of Motor Proteins and the Cytoskeleton (Sinauer, Sunderland, MA, 2001).

[2] S. Leibler and D. A. Huse, J. Cell Biol. 121, 1357 (1993).

[3] R. D. Vale, D. R. Soll, and I. R. Gibbons, Cell 59, 915 (1989).

[4] F. Jülicher and J. Prost, Phys. Rev. Lett. 75, 2618 (1995).

[5] A. Vilfan, E. Frey, and F. Schwabl, Eur. Phys. J. B 3, 535 (1998).

[6] C. Leduc et al., Proc. Natl. Acad. Sci. U.S.A. 101, 17096 (2004).

[7] I. Derényi, F. Jülicher, and J. Prost, Phys. Rev. Lett. 88, 238101 (2002).

[8] R. Lipowsky, S. Klumpp, and T. M. Nieuwenhuizen, Phys. Rev. Lett. 87, 108101 (2001).

[9] A. Parmeggiani, T. Franosch, and E. Frey, Phys. Rev. Lett. 90, 086601 (2003).

[10] M. R. Evans, Europhys. Lett. 36, 13 (1996).

[11] We assume for simplicity that the $N$-motor problem can be described with the same rates as the two motor case: we ignore higher order correlations in the rates.

[12] N. G. Van Kampen, Stochastic Processes in Physics and Chemistry (North-Holland, Amsterdam, 2004).

[13] M.E. J. Newman and G. T. Barkema, Monte Carlo Methods in Statistical Physics (Clarendon, Oxford, 1999).

[14] S. Klumpp and R. Lipowsky, Proc. Natl. Acad. Sci. U.S.A. 102, 17284 (2005).

[15] F. Jülicher, A. Ajdari, and J. Prost, Rev. Mod. Phys. 69, 1269 (1997).

[16] J. Snider et al., Proc. Natl. Acad. Sci. U.S.A. 101, 13204 (2004).

[17] We use values of the kinetic parameters approximately equal to those measured experimentally for conventional kinesin [18].

[18] N. J. Carter and R. A. Cross, Nature (London) 435, 308 (2005). 